Research Article

\title{
The PBAR Protocol: Antipsychotic Medication Reduction in the Nursing Home
}

Melodee Harris ${ }^{1,}{ }^{*}$, Priya Mendiratta ${ }^{2}$, Gohar Azhar ${ }^{2}$, Ruofei Du ${ }^{3}$, Jing Jin ${ }^{3}$

1. University of Arkansas for Medical Sciences College of Nursing, US; E-Mail: harrismelodee@uams.edu

2. University of Arkansas for Medical Sciences Reynolds Insitute on Aging, US; E-Mails: PMendiratta@uams.edu; AzharGohar@uams.edu

3. University of Arkansas for Medical Sciences College of Public Health Department of Biostatistics, US; E-Mails: RDu@uams.edu; JJIN@uams.edu

* Correspondence: Melodee Harris; E-Mail: harrismelodee@uams.edu

Academic Editor: Ladislav Volicer

Special Issue: Advanced Dementia: Enjoy It or Avoid It?

OBM Geriatrics

2021, volume 5, issue 2

doi:10.21926/obm.geriatr.2102172
Received: November 24, 2020

Accepted: May 20, 2021

Published: June 15, 2021

\begin{abstract}
Neuropsychiatric symptoms (NPS) occur in almost all persons living with dementia. Antipsychotic medications are often prescribed inappropriately for persons living with dementia who experience NPS. Adverse side effects of antipsychotic medications include falls, sedation, cognitive dysfunction, prolonged QTc interval, extrapyramidal side effects, cerebrovascular events, metabolic changes, infection, and mortality. The purpose of this descriptive quality improvement project was to test the feasibility and preliminary effectiveness of using the Psychiatric Background Assessment Recommendation (PBAR) Interprofessional Communication Tool (ICT). The PBAR-ICT was adapted from the Situation Background Assessment Recommendation (SBAR) Protocol by the Principal Investigator and was used to report critical NPS in the nursing home. After approval from the university institutional review board, an education program on identifying NPS was provided to the nursing staff. The education program focused on using terminology to destigmatize and
\end{abstract}

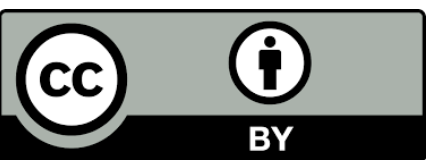


standardize NPS. Training was also provided on how to use the PBAR-ICT. A six-month retrospective and prospective chart review were completed on all charts documenting an antipsychotic medication prescription. Medication changes were analyzed to determine successful or unsuccessful deprescribing by comparing data on pre and post PBAR-ICT implementation. Chart diagnoses $(n=104)$ included dementia $(n=55)$, schizophrenia/bipolar disorder $(n=14)$, schizophrenia/bipolar disorder plus dementia $(n=23)$ or no documented diagnoses $(n=12)$. Relative to pre PBAR-ICT implementation for all charts $(n=104)$, results on post PBAR-ICT implementation estimated 1.57 times higher odds $(p=0.003)$ for decreasing and/or discontinuing antipsychotic medications. Outcomes on post PBAR-ICT implementation were analyzed by diagnoses. When compared with the schizophrenia/bipolar disorder plus the dementia group, successful discontinued/decreased antipsychotic medications were statistically significant $(p=0.015)$ for the dementia only group. Results of this quality improvement project demonstrated the feasibility of implementing the PBAR-ICT in the nursing home. The PBAR-ICT is an interprofessional communication tool that can play a role in communicating NPS to discontinue and decrease antipsychotic medications.

\section{Keywords}

Neuropsychiatric symptoms; antipsychotic medications; dementia; schizophrenia; bipolar disorder

\section{Introduction}

Americans are twice as fearful of losing mental capacity than losing physical health [1]. According to the United States Census Bureau, there are 5.8 million people living with a diagnosis of dementia [2]. For persons living with dementia, memory loss is further complicated by neuropsychiatric symptoms [3, 4].

Neuropsychiatric symptoms are associated with severity of dementia and contribute to early nursing home placement [5]. It is estimated that 47.8 percent of all nursing home residents have a diagnosis of Alzheimer's dementia and other dementias [6]. In a study of residents who were directly admitted from home to a nursing home, 1.63 percent $(p=.53)$ had a mental health diagnosis and 16.36 percent $(p<.001)$ had behavioral problems [7]. Incidence rates of any one neuropsychiatric symptom is between 20-56 percent [8]. The cumulative prevalence rate of any one neuropsychiatric symptom occurs in 48 to 97 percent of persons living with dementia in the nursing home [8].

Neuropsychiatric symptoms are also referred to as behavioral and psychological symptoms of dementia (BPSD) and occur in almost all persons living with dementia [9-11], placing them at risk for polypharmacy [12], progression to severe disease [13] and unnecessary hospitalizations [12]. Terminology for neuropsychiatric symptoms is inconsistent [14]. In 1994, the International Psychogeriatrics Association (IPA) Consensus Group [15] proposed this definition, "The term behavioral disturbances should be replaced by the term behavioral and psychological symptoms of dementia (BPSD), defined as: symptoms of disturbed perception, thought content, mood or behavior that frequently occur in patients with dementia" [16]. The IPA categorizes neuropsychiatric symptoms as either behavioral or psychological symptoms [15]. Healthcare providers must 
implement innovative strategies to manage neuropsychiatric symptoms, deprescribe inappropriate antipsychotic medications, advance person-centered care and promote resiliency for older adults living with dementia. However, before such strategies can be implemented, there must an operational definition and standardization of neuropsychiatric symptoms [14].

Antipsychotic medications are frequently prescribed for persons living with dementia who experience neuropsychiatric symptoms $[17,18]$. The American Psychological Association (APA) published practice guidelines and varying evidence for benefits and risks of antipsychotic medication use in persons living with dementia [17]. The APA recommends that before nonemergency use of antipsychotic medications that clinicians examine the type of dementia and weigh risks for adverse side effects including cognitive dysfunction, hypotension and dizziness resulting in falls, sedation, prolonged QTc interval, extrapyramidal side effects, increased cerebrovascular events, metabolic changes, infection and increased mortality $[17,18]$.

In spite of black box warnings, antipsychotic medications are inappropriately prescribed for persons living with dementia in the nursing home. The national rate for antipsychotic medication use decreased from $23.9 \%$ in 2011 to $14.1 \%$ in 2020 [19, 20]. One study [20] examined initiatives of the National Partnership for Improving Dementia Care. Among residents, nursing home staff, prescribers and informal caregivers, gaps were identified in application of knowledge on antipsychotic medications. On one hand, nursing home staff and prescribers agreed that antipsychotic medications impose significant risks for persons living with dementia. On the other hand, residents, nursing home staff and families responded to neuropsychiatric symptoms with requests to prescribers for antipsychotic medications [20]. In addition to nursing homes staff, families also requested education on dementia. Nursing assistants played an important role in improving person-centered care and individualizing practices for neuropsychiatric symptoms. Particularly relevant to our study, results showed that clear communication between staff and prescribers was an important outcome [20]. To further decrease the inappropriate use of antipsychotic medications in persons living with dementia, strategies are needed to standardize communication of neuropsychiatric symptoms and promote safe medication deprescribing.

The purpose of this quality improvement project was to test the feasibility and preliminary effectiveness of using the Psychiatric Background Assessment Recommendation (PBAR) Interprofessional Communication Tool (ICT; Figure 1). The PBAR-ICT Protocol was adapted from the Situation Background Assessment Recommendation (SBAR) Protocol [21] by the Principal Investigator and was used to report critical neuropsychiatric situations in the nursing home. This is the first time an interprofessional tool to specifically address neuropsychiatric symptoms was implemented in a nursing home as a strategy for antipsychotic medication reduction. 


\section{Patient Name}

\begin{tabular}{|c|c|}
\hline & 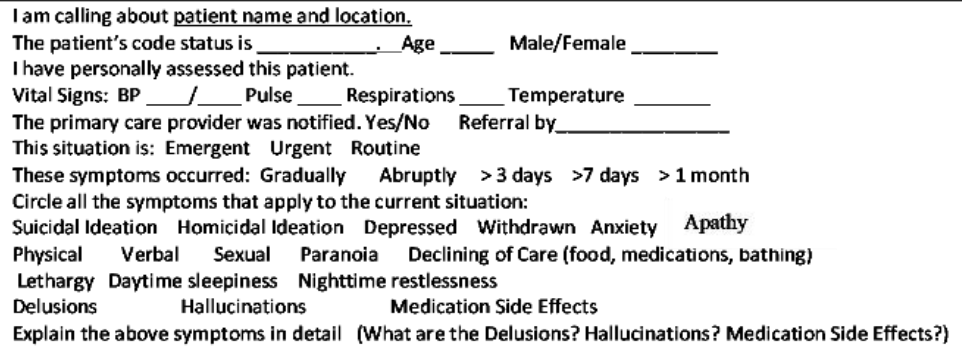 \\
\hline & $\begin{array}{l}\text { Background: } \\
\text { The patient has: } \\
\text { Dementia Depression Anxiety Schizophrenia Bipolar Disorder Delirium } \\
\text { Allergies: } \\
\text { Medications (Dose/Frequency): } \\
\text { Antidepressants } \\
\text { Anxiolytics } \\
\text { Antipsychotics } \\
\text { Mood Stabilizer } \\
\text { Estrogen } \\
\text { New Medications: } \\
\text { Recent Hospitalizations Location/Reason: }\end{array}$ \\
\hline & $\begin{array}{l}\text { Assessment } \\
\text { This is what I think the problem is: } \\
\text { The problem seems to be } \\
\text { I am not sure what the problem is, but the patient is deteriorating. } \\
\text { The patient is unstable and we may need to do something. }\end{array}$ \\
\hline & $\begin{array}{l}\text { Recommendation } \\
\text { I suggest or request that you } \\
\text { Transfer to ER } \\
\text { Transfer to geropsychiatric inpatient evaluation. } \\
\text { Medication adjustment in facility. Order UA C/S } 72 \text { hours only if symptoms persist } \\
\text { Are there any tests needed? Order: BP BMP CBC Depakote level Lithium level } \\
\text { If a change in treatment is ordered, then ask: } \\
\text { What follow up care is needed? } \\
\text { How long do you expect this problem to last? } \\
\text { If the patient does not improve, how do you want us to follow up? }\end{array}$ \\
\hline
\end{tabular}

Reporter Name/Date: Reported to:

This PBAR tool is adapted from the SBAR Kaiser Permanente. Revised by Melodee Harris, PhD, RN, FAAN. Use only with permission. Email harrismelodee@uams.edu for permission or recommendations.

Figure 1 PBAR report about a critical geropsychiatric situation.

\section{Methods}

\subsection{Design and Sample}

This is a descriptive study of a quality improvement project to test the feasibility of the PBAR-ICT protocol in five non-profit nursing homes in the Southeastern United States. There was a combined occupancy of 717 beds. The estimated average number of beds was 143 with few private rooms. Demographics, admission criteria, socioeconomic status and Medicare beds were similar. Two nursing homes had designated behavioral units. The nursing homes were required to be in compliance with recommended minimum staffing requirements based on 1:5 residents on day shift, 1:10 residents on evening shift and 1:15 residents on night shift [22]. 
Medical directors and directors of nursing agreed to participate in the quality improvement project. The quality improvement project involved providing education on neuropsychiatric symptoms, using the PBAR-ICT to report neuropsychiatric symptoms, and collecting chart data on antipsychotic medication reduction before and after education and implementation of the PBARICT.

After approval from the university institutional review board, an education program on neuropsychiatric symptoms and antipsychotic medications was provided to nurses and nursing assistants. See Figure 2. The same education program on dementia and neuropsychiatric symptoms was presented to nursing staff at each nursing home. Nurses and nursing assistants were given a laminated card that was used as a reminder about diagnoses, medications, inconsistent staffing or other possible reasons for changes in neuropsychiatric symptoms. Although they were familiar with the project, none of the medical directors or primary care physicians participated in the education programs.

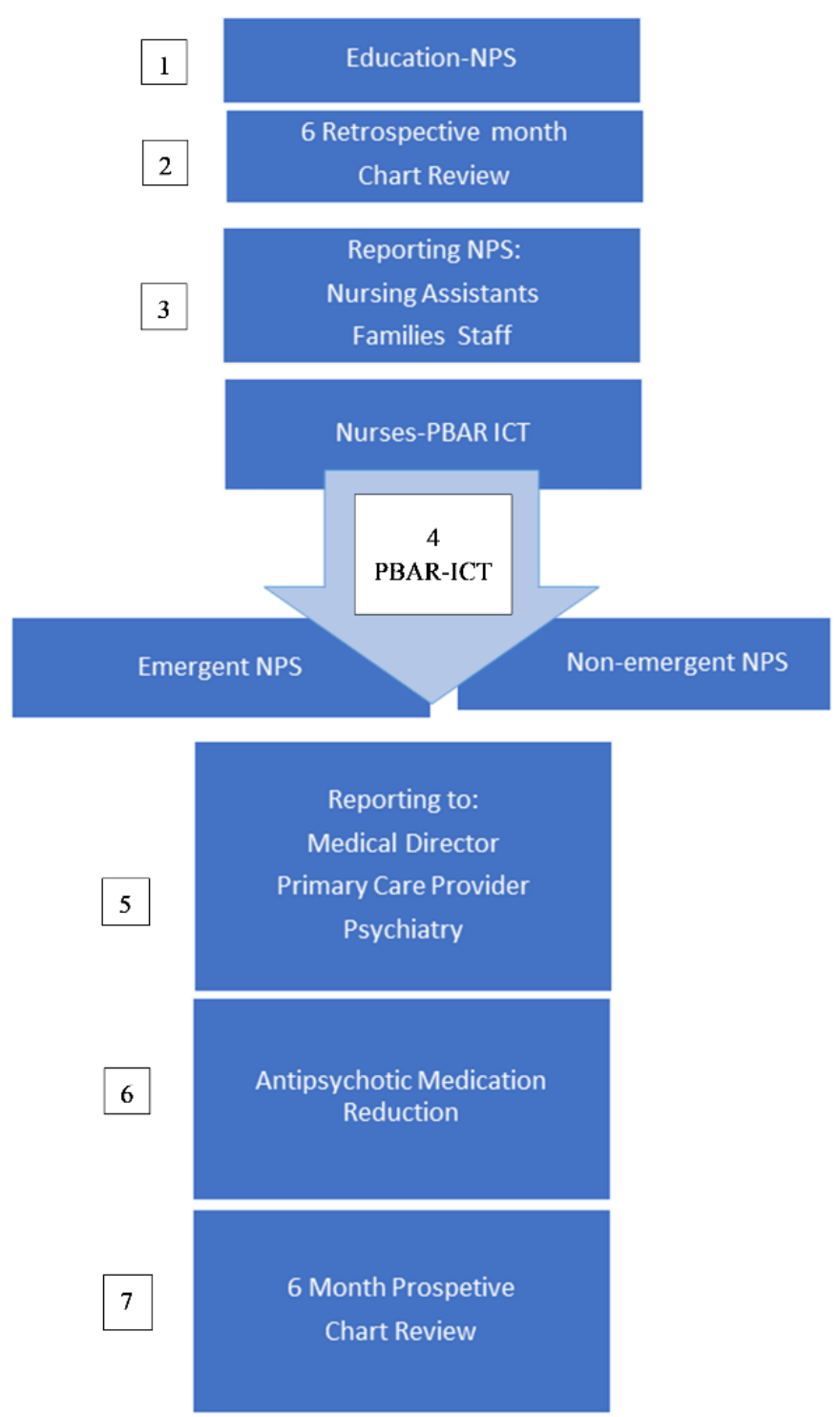

Figure 2 Intervention protocol. 
Education on interprofessional communication and the PBAR-ICT was provided to each nursing home. Nurses were specifically trained to complete the PBAR-ICT and report critical neuropsychiatric symptoms to providers. The PBAR-ICT is similar to the Symptom Background Assessment Recommendation (SBAR) Tool. The SBAR Tool is used in nursing homes to report changes in condition [21] but does not address neuropsychiatric symptoms specific to nursing home residents living with dementia or mental illnesses. Critical neuropsychiatric symptoms were discussed with providers over the phone or placed in a notebook to communicate timely, urgent needs that could be addressed on routine rounding by the provider.

After agreeing to the education and protocol implementation, the director of nursing provided a list of nursing home residents who were prescribed an antipsychotic medication. The principal investigator collected the data or designated and trained a nurse on how to perform six-month retrospective and prospective chart reviews on the variables. The six-month retrospective chart reviews captured data before implementing the PBAR-ICT. A six-month prospective chart review was also used to collect the data on protocol implementation. The principal investigator followed up by reviewing the chart data.

Chart diagnoses of dementia, schizophrenia and bipolar disorder were collected. Neuropsychiatric symptom clusters for affective (depression and anxiety), psychosis (hallucinations and delusions), refusal of care, and symptoms of verbal and physical agitation/aggression, antipsychotic medications and prescribing patterns were collected and analyzed. Nonpharmacological interventions were also recorded. The Minimum Data Set was used to clarify information on variables.

A six-month retrospective and prospective chart review was completed to track increased, decreased and discontinued antipsychotic medications. The response to medication changes was collected and analyzed to determine successful or unsuccessful medications pre and post protocol implementation. According to deprescribing guidelines, decreased or discontinued medication within three months after initiation was determined as criteria for successful medication adjustments $[23,24]$.

Six months of retrospective chart data were extracted from 104 charts before the protocol implementation. The selected charts served as their own controls to test the effectiveness of the education program when compared to 6-month prospective chart review after implementation of the PBAR-ICT. Inclusion criteria for review were all charts from each nursing home with at least one prescribed antipsychotic medication. Data were collected from 2014-2017.

The PBAR-ICT was implemented to report critical neuropsychiatric symptoms. Nursing assistants reported neuropsychiatric symptoms to the nurses. Nurses used the PBAR-ICT to categorize neuropsychiatric symptoms as emergent or nonemergent and report these symptoms to the appropriate provider. Specific neuropsychiatric symptoms that required possible medication changes were reported to providers in order to avoid physical, functional or cognitive decline, exacerbation of neuropsychiatric symptoms or unnecessary hospitalization.

Providers were the medical directors and primary care providers. All five nursing homes had access to either a geriatrician, psychiatrist or a geropsychiatric advanced practice nurses. Investigators on this quality improvement project were represented at each of the nursing homes, but were not always involved in all decision-making for prescribing. Nurses reported neuropsychiatric symptoms to the resident's chosen provider. Some residents were managed by outside clinic providers and some prescribers were associated with hospital admissions. 


\section{Statistical Analysis}

A logistic regression was fitted with one of the binary outcomes (number of either total discontinued or decreased medications at post-protocol implementation greater than preimplementation) against age, gender, nursing home and the diagnosis. Age was treated as a continuous covariate, and the others were treated as categorical covariates. Wald tests imply insignificant effects of age $(p=0.75)$, gender $(p=0.52)$ and nursing home $(p=0.31)$ and thus those covariates were not further considered in the major analysis.

Limited by a small sample size $(\mathrm{N}=104)$, the protocol implementation effect (post vs. pre-protocol implementation) were specifically examined either for all data, within a subgroup of a covariate, or by a comparison between subgroups of a covariate. The Exact McNemar approach was employed for analyzing a contingency table with pre- and post- measures collected from the same participants at the pre- and post- time points. The Fisher's or Chi-square Exact tests were applied for the comparison between subgroups.

\section{Results}

\subsection{Demographics}

The demographics for nursing personnel $(n=136)$ across the five nursing homes (Table 1$)$ shows that most nursing assistants, licensed practical nurses and registered nurses were females between 22-65 years old. The majority were employed full time, with more than five years of experience and worked in the nursing home for less than three years. In general, participating nursing homes variables were homogenous. 
Table 1 Demographics of the nursing personnel in the participated nursing homes (NHs).

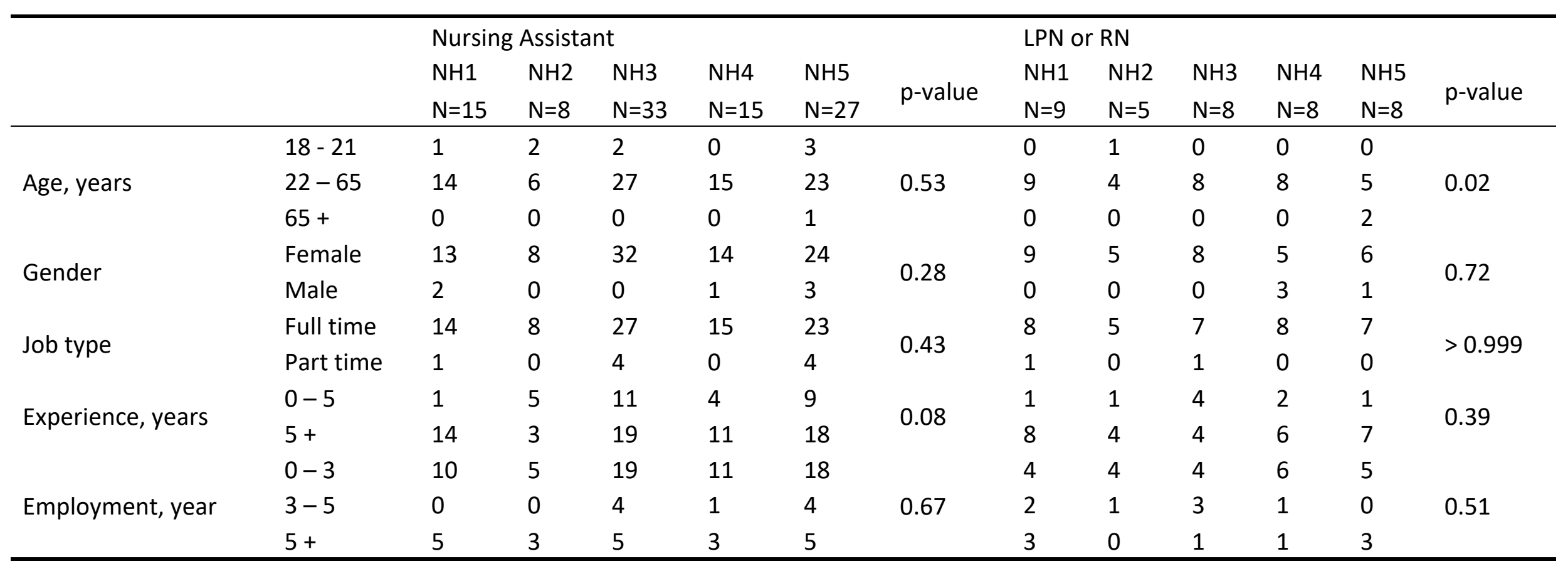

Note: The distributions of baseline variables were compared by Chi-square tests, and the p-values were obtained using Monte Carlo simulations for small sample size tables. Missing values were not presented in table, and not included in the tests. 
This sample of charts $(n=104)$ with prescribed antipsychotic medications resulted in 68 females (65.4\%) and 36 males (34.6\%) with a mean age of 74 years old (Table 2). There were 55 chart diagnoses of dementia only, 14 had a diagnosis of schizophrenia or bipolar disorder only, 23 had a chart diagnoses of dementia plus schizophrenia or bipolar disorder and 12 charts were without any recorded chart diagnoses (Table 2).

Table 2 Demographics-Chart Data.

\begin{tabular}{llll}
\hline Var & Value & $\mathrm{N}=104$ & Mean (Std) / Prop. \% \\
\hline Age & (integer) & 101 & $74.2(14.7)$ \\
Gender & Female & 68 & 65.4 \\
& Male & 36 & 34.6 \\
& dementia diagnoses only & 55 & 52.9 \\
Diagnosis & schizophrenia or bipolar disorder & 23 & 22.1 \\
& plus dementia diagnoses & & \\
& Schizophrenia or bipolar disorder & 14 & 13.5 \\
& diagnoses only & 12 & 11.5 \\
& no chart diagnoses & 51.9 \\
Medication ${ }^{\text {a }}$ & Quetiapine & 54 & 22.1 \\
& Olanzapine & 23 & 21.2 \\
& Risperidone & 22 & 14.4 \\
& Haloperidol & 15 & 5.8 \\
& Aripiprazole & 6 & 2.9 \\
& Other & 3 & 44.2 \\
Neuropsychiatric & Hallucination or Delusion & 46 & 38.5 \\
symptom & Anxiety or depression & 40 & \\
& physical/Verbal & & \\
& agitation/aggression or refusal of & 10 & 9.6 \\
& care & 8 & 7.7 \\
\hline
\end{tabular}

${ }^{a}$ Since the Medication categories are not exclusive to one another, the sum of Props. \% for the Medications $>1$.

\subsection{Analysis Results}

By gender, there was no statistical significance for any specific neurocognitive symptom. An analysis of prescribed medications includes quetiapine $51.9 \%(n=54)$, olanzapine $22.1 \%(n=23)$, risperidone $21.2 \%(n=22)$, haloperidol $14.4 \%(n=15)$, aripiprazole $5.8 \%(n=6)$ and $2.9 \%(n=3)$ on thorazine, fluphenazine or ziprasidone (Table 2). A patient could have been on more than one medication. There was no statistical significance as a result of the protocol implementation for reducing or increasing the number of falls due to medication adjustments.

Data were collected on neuropsychiatric symptom clusters, prescribing patterns on antipsychotic medications for increases, decreases and discontinued medications pre and post intervention. An overall and specific analysis was done on increased, decreased and discontinued antipsychotic medications. 
For all participants, there were no statistically significant differences $(p=0.901)$ pre and post implementation on increases in antipsychotic medications (Table 3). For all participants and relative to pre protocol implementation, post implementation outcome results showed an estimated 1.57 times higher odds $(p=0.003$ ) on decreased and/or discontinued antipsychotic medications (Table 4).

Table 3 Increased Medications. All participants:

\begin{tabular}{llllll}
\hline Pre- & $\begin{array}{l}\text { Post- } \\
\text { No }(=0)\end{array}$ & Yes $(>0)$ & Tot & OR $(95 \% \mathrm{Cl})$ & P value \\
\hline No $(=0)$ & 24 & 33 & 57 & & \\
Yes $(>0)$ & 31 & 16 & 47 & $1.06(0.63,1.8)$ & 0.901 \\
Tot & 55 & 49 & 104 & & \\
\hline
\end{tabular}

Table 4 Total discontinued or decreased medications. All participants:

\begin{tabular}{llllll}
\hline Pre- & Post- & & & OR $(95 \% \mathrm{Cl})$ & P value \\
& No $(=0)$ & Yes $(>0)$ & Tot & & \\
\hline No $(=0)$ & 29 & 36 & 65 & & $0.003^{*}$ \\
Yes $(>0)$ & 14 & 25 & 39 & $2.57(1.35,5.16)$ & \\
Tot & 43 & 61 & 104 & & \\
\hline
\end{tabular}

Results were analyzed on post protocol implementation by diagnosis. When compared with the schizophrenia/bipolar plus dementia group, the dementia only group $(p=0.056)$ trended toward more successfully discontinued antipsychotic medications (Table 5). When compared with the schizophrenia/bipolar plus dementia group (Table 6), the dementia only group experienced more successfully decreased or discontinued medications post protocol implementation $(p=0.015)$. 
Table 5 Discontinued medications number of total discontinued medications greater at post than at pre-protocol implementation. By diagnosis:

\begin{tabular}{|c|c|c|c|c|c|}
\hline Diagnosis & post $>$ pre & post $\leq$ pre & Prop. $(95 \% \mathrm{Cl})$ & Effect of Diagnosis on Prop. & $\begin{array}{l}\text { dementia only vs. } \\
\text { schizophrenia/bipolar only plus } \\
\text { dementia }\end{array}$ \\
\hline dementia only & 15 & 40 & $0.27(0.16,0.41)$ & \multirow{3}{*}{$p=0.156$} & \multirow{3}{*}{$\begin{array}{l}\text { Prop diff, } \mathrm{Cl}: 0.16(0.01,0.32) \\
p=0.056\end{array}$} \\
\hline SBP only or/and Dementia & 4 & 33 & $0.11(0.03,0.25)$ & & \\
\hline Neither SBP nor Dementia & 3 & 9 & $0.25(0.05,0.57)$ & & \\
\hline
\end{tabular}

Table 6 Number of either total discontinued or decreased medications greater at post- than at pre-protocol implementation. By diagnosis:

\begin{tabular}{|c|c|c|c|c|c|}
\hline Diagnosis & post $>$ pre & post $\leq$ pre & Prop. $(95 \% \mathrm{Cl})$ & Effect of Diagnosis on Prop. & $\begin{array}{l}\text { dementia only vs. } \\
\text { schizophrenia/bipolar only plus } \\
\text { dementia }\end{array}$ \\
\hline Dementia only & 29 & 26 & $0.53(0.39,0.66)$ & \multirow{3}{*}{$p=0.045^{*}$} & \multirow{3}{*}{$\begin{array}{l}\text { Prop diff, } \mathrm{Cl}: 0.26(0.062,0.452) \\
\mathrm{p}=0.015^{*}\end{array}$} \\
\hline SBP only or/and Dementia & 10 & 27 & $0.27(0.14,0.44)$ & & \\
\hline Neither SBP nor Dementia & 6 & 6 & $0.50(0.21,0.79)$ & & \\
\hline
\end{tabular}


Any combination of post-protocol implementation on successfully decreased or discontinued medications could not be attributed to any specific antipsychotic medication. In summary, the PBAR-ICT protocol was effective at the 0.05 significance level on outcomes for discontinuing or decreasing antipsychotic medications.

\section{Discussion}

Terminology, tools and medication adjustments were the basic considerations for this quality improvement project. Consistent, operational definitions are critical for tools that are used to accurately describe, measure and communicate neuropsychiatric symptoms [25]. In this project, the terminology used in the PBAR-ICT communicated neuropsychiatric symptoms and contributed to successful medication adjustments.

One systematic approach for managing neuropsychiatric symptoms is the DICE Model. The DICE acronym includes: Describing neuropsychiatric symptoms, Investigating the cause, Collaboration to create an intervention, implementing a treatment plan and then Evaluating the safety and efficacy of the intervention. Similar to other strategies, the DICE Model is based on the supposition that the neuropsychiatric symptom has been recognized and called to the provider's attention [26]. This quality improvement project focused on communicating accurate terminology that is foundational to methods such as the DICE Model that depend upon accurate recognition and communication of neuropsychiatric symptoms. The PBAR-ICT is an interprofessional communication tool that can be integrated into models for the assessment, treatment and management of neuropsychiatric symptoms.

There is controversy associated with the terminology for neuropsychiatric symptoms [14]. The education component of this quality improvement project emphasized the need to standardize terminology and prevent stigma in persons with living dementia. Neuropsychiatric symptoms are often referred to as "behaviors" or "agitation" [25, 27]. "Refusal or resistance of care" is another subjective term $[25,27,28]$. Using this terminology promotes stigma because it implicates the person living with dementia instead of the considering the context of the situation or consequence of the environment $[28,29]$. These terms are subjective with an inherent negative connotation that inaccurately describes the disease process of dementia. Neuropsychiatric symptoms are the result of a trajectory of disease progression and are not within the control of anyone victimized by the disease [29]. Interprofessional communication of neuropsychiatric symptoms is critical for treatment, decision-making and safe delivery of care.

Priority must be given to terminology that will prevent stigma and promote a culture of wellbeing for persons living with dementia. Over time, experts in neuropsychiatric symptoms have addressed the importance of developing a frame of reference for neuropsychiatric symptoms. Agitation and aggression are combined in the Cohen-Mansfield Inventory [30]. A Consensus Group from the International Psychogeriatric Association developed a provisional consensus clinical and research definition of agitation [31]. Studies have distinguished agitation, aggression [28, 29, 32] and resistance of care [28]. In real-life, terminology tends to lean toward subjectivity.

One study on the impact of initiatives from the Partnership for Improving Dementia Care identifies that clear communication is important to antipsychotic medication reduction. Therefore, education for the PBAR Protocol focused on more accurately communicating neuropsychiatric 
symptoms. Fairly representing persons living with dementia using an interprofessional approach is important for antipsychotic medication reduction and improving dementia care [20].

The PBAR-ICT replaces the term "behavior" or "agitation" with the term "symptom." Refusal of care did not follow the Minimum Data Set definition [28]. Instead, responses for the PBAR-ICT required clarification with possible explanations for declining mediations, meals, bathing or other care. Specific delusions and hallucinations were also identified on the PBAR-ICT. Our results were consistent with the literature that neuropsychiatric symptoms are rarely a single symptom [4]. For example, symptoms of agitation overlap with refusal of care [28].

It is important to use tools that are culturally appropriate and population-focused [33]. Specific tools are needed that make a clear distinction between neuropsychiatric symptoms [25, 28, 32, 33]. The Scale for Observation of Agitation in Persons with Dementia of the Alzheimer's Type (SOAPD) is a measurement tool for agitation in persons with dementia [27]. The Neuropsychiatric Inventory [34] and Cohen Mansfield Inventory [30] are widely used in the measurement of neuropsychiatric symptoms. The PBAR-ICT was modified from the SBAR Tool to communicate psychological symptoms that apply to the person living with dementia. Because the population focus for this quality improvement project was older adults, neuropsychiatric symptoms were termed as geropsychiatric symptoms.

The initial PBAR-ICT was meant to evolve in response to recommendations throughout the project. After considering input from nursing staff, several changes were made to the initial PBARICT. One addition to the PBAR-ICT included collecting a urinalysis 72 hours after the onset of increased symptoms. This change was made to promote antibiotic stewardship. Symptoms on sleep were also added to the final version of the PBAR-ICT (Figure 1).

The implications for addressing terminology and the PBAR-ICT were reflected in results on medication adjustments and recording neuropsychiatric symptoms. In our project, quetiapine was more widely used than other antipsychotic medications, possibly due to decreased mortality when compared with risperidone and haloperidol [35].

When outcomes were analyzed by diagnosis, there were more successful medication adjustments in the dementia only group compared with the schizophrenia and bipolar plus dementia group [20]. This is consistent with literature and guidelines that recommend tapering and discontinuing antipsychotic medications in persons living with dementia [23].

Guidelines support the use of antipsychotic medications for older adults with severe and persistent mental illness such as lifelong schizophrenia [17, 24]. In our project across all diagnoses, hallucinations and delusions was the most common reason for prescribing an antipsychotic mediation.

There were almost as many documented hallucinations or delusions ( $n=46 ; 44.2 \%$ ) as depression and anxiety ( $n=40 ; 38.4 \%)$ clusters; all of which are common neuropsychiatric symptoms [10]. Our findings were consistent with the literature. Depression is one of the most common neuropsychiatric symptoms in persons living with mild cognitive impairment and early dementia [10]. If nonpharmacological interventions fail in the treatment of verbal symptoms, antidepressants medications may be prescribed to prevent combativeness [36] and decrease neuropsychiatric symptoms [37]. Depression can herald the transition from mild cognitive impairment to dementia $[4,38]$ while delusions and hallucinations typically appear after the onset of dementia [38]. 


\section{Limitations}

This project was limited by the small sample size, lack of a control group and randomization. Although the Minimum Data Set was used to confirm data collection, it was not the only source of information for data collection in this project. Due to inconsistent terminology and staff training, the Minimum Data Set is not always a reliable source of information. Knowledge gaps such as distinguishing between hallucinations and delusions, depression and anxiety or delirium and schizophrenia presented challenges for specific measurements of neuropsychiatric symptoms. In addition, the success of the project could have been propelled forward due to mandatory regulation by Centers for Medicare and Medicaid Services to reduce antipsychotic medications in nursing homes.

In the future, implementing the PBAR-ICT intervention could be strengthened more by what was not documented in this study. The subtype and severity of dementia should be measured. Variables on sleep and apathy were not addressed throughout the project and were added to the PBAR-ICT. Agitation, aggression and resistive/refusal of care should be clearly distinguished in future projects. Data were collected and analyzed on antipsychotic medications, but not mood stabilizers, antidepressants, anxiolytics and hypnotic medications. It would be important to know the associated neuropsychiatric symptoms that influenced these prescribing patterns.

Importantly, nurses did not track the numbers of completed PBAR-ICT documents. All nursing homes implemented the PBAR-ICT. One nursing home filed 19 PBAR documents, but this documentation was inconsistent. In addition, although the data collection form specified identifying nonpharmacological interventions, other than general statements such as "calm reassurance" or "redirection" there was little to no documentation of specific nonpharmacological interventions. Finally, quality of life was not measured. Although initiatives such as the Partnership for Improving Dementia Care are successful and contributed to the profound reduction in antipsychotic medication reduction in nursing homes, quality of life has not been studied with the same intensity [20]. A section on the PBAR-ICT could make recommendations for individual interventions to improve quality of life.

\section{Conclusion and Future Research}

The results of this quality improvement project demonstrated preliminary success in effectively communicating neuropsychiatric symptoms. Effective communication of neuropsychiatric symptoms is reflected in the results on successful decreases and discontinued antipsychotic medications. Clearly identifying neuropsychiatric symptoms is not only important for antipsychotic medication reduction, but for identifying disease process and progression. Neuropsychiatric symptoms do not predict dementia subtypes, but some neuropsychiatric symptoms are biomarkers for predicting incident dementia [39].

Future projects should consider early identification of neuropsychiatric symptoms to expedite appropriate treatment that can promote greater autonomy and prevent early nursing home placement. The National Partnership for Improving Dementia Care has significantly influenced decreasing antipsychotic medication use in nursing homes. However, the risk of untreated neuropsychiatric symptoms has not been widely studied. Strategies and tools to effectively document nonpharmacological interventions are also needed. Decreases in neuropsychiatric 
symptoms due to nonpharmacological interventions and improvement in quality of life is the goal of antipsychotic medication reduction and should be studied in nursing home residents with the same level of priority as decreasing medications.

Older adults are fearful of living with dementia. Using accurate terminology that is free of stigma can go a long way to diminish fears. Health care professionals owe it to this incredible generation to continuously implement innovative strategies that will improve the quality of life for persons with dementia.

\section{Author Contributions}

All authors reviewed the results and approved of the final manuscript.

Statement of authorship was modified from the following:

\section{Criteria for authorship is consist with International Committee of Medical Journal Editors (ICMJE) and recommended by the Committee on Publication Ethics (COPE).}

http://www.icmje.org/recommendations/browse/roles-and-responsibilities/defining-the-roleof-authors-and-contributors.html

\section{https://publicationethics.org}

The following is from http://onlinepubs.trb.org/onlinepubs/TRREM/AuthorContribution.pdf

MELODEE HARRIS: 1. made a substantial contribution to the concept or design, acquisition, analysis, and interpretation of data for the article; AND 2.drafted the article and revised it critically for important intellectual content; AND 3. approved the version to be published; AND 4. agreed to be accountable for all aspects of the work in ensuring that questions related to the accuracy or integrity of any part of the work are appropriately investigated and resolved.

PRIYA MENDIRATTA: 1 . made a substantial contribution to the concept and design of the article; or the acquisition, analysis, or interpretation of data for the article; AND 2. drafted the article or revised it critically for important intellectual content; AND 3. approved the version to be published; AND 4. agreed to be accountable for all aspects of the work in ensuring that questions related to the accuracy or integrity of any part of the work are appropriately investigated and resolved

GOHAR AZHAR: 1. made a substantial contribution to the concept and design of the article; or the acquisition, analysis, or interpretation of data for the article; AND 2. drafted the article or revised it critically for important intellectual content; AND 3. approved the version to be published; AND 4. agreed to be accountable for all aspects of the work in ensuring that questions related to the accuracy or integrity of any part of the work are appropriately investigated and resolved

RUOFEI DU: 1. performed the statistical data analyses. 2. responsible for integrity of analysis approaches and results. 3. wrote the statistical analysis section, provided the tables and assisted in interpretation of the analysis results. 4. read and approved the version to be published.

JING JIN: 1. performed the statistical data analyses. 2. wrote the statistical analysis section, provided the tables and assisted in interpretation of the analysis results. 3. read and approved the version to be published.

\section{Funding}

1. Gerontological Advanced Practice Nursing Association Foundation;

2. University of Arkansas for Medical Sciences College of Nursing Intramural Grant. 


\section{Competing Interests}

The authors have declared that no competing interests exist.

\section{References}

1. At a glance Alzheimer's disease. Atlanta, Georgia: Center for Disease Control and Prevention; 2018. Available from: https://www.cdc.gov/aging/publications/aag/alzheimers.html.

2. Hebert LE, Weuve J, Scherr PA, Evans DA. Alzheimer disease in the United States (2010-2050) estimated using the 2010 census. Neurology. 2013; 80: 1778-1783.

3. Tschanz JT, Corcoran CD, Schwartz S, Treiber K, Green RC, Norton MC, et al. Progression of cognitive, functional, and neuropsychiatric symptom domains in a population cohort with Alzheimer dementia: The cache county dementia progression study. Am J Geriatr Psychiatry. 2011; 19: 532-542.

4. Lanctôt KL, Amatniek J, Ancoli-Israel S, Arnold SE, Ballard C, Cohen-Mansfield J, et al. Neuropsychiatric signs and symptoms of Alzheimer's disease: New treatment paradigms. Alzheimers Dement Trans Res Clin Interv. 2017; 3, 440-449.

5. Chan DC, Kasper JD, Black BS, Rabins PV. Presence of behavioral and psychological symptoms predicts nursing home placement in community-dwelling elders with cognitive impairment in univariate but not multivariate analysis. J Gerontol A. 2003; 58: 548-554.

6. Harris-Kojetin L, Sengupta M, Lendon JP, Rome V, Valverde R, Caffrey C. Long-term care providers and services users in the United States, 2015-2016. Hyattsville: National Center for Health Statistics: Vital and Health Statistics; 2019; 2No.2019-1427.

7. Holupa A, Hyer K, Meng HD, Volicer L. Profile of nursing home residents admitted directly from home. J Am Med Dir Assoc. 2017; 18: 131-137.

8. Selbæk G, Engedal K, Bergh S. The prevalence and course of neuropsychiatric symptoms in nursing home patients with dementia: A systematic review. J Am Med Dir Assoc. 2013; 14: 161169.

9. Phan SV, Osae S, Morgan JC, Inyang M, Fagan SC. Neuropsychiatric symptoms in dementia: Considerations for pharmacotherapy in the USA. Drugs R D. 2019; 19: 93-115.

10. Lyketsos CG, Carrillo MC, Ryan JM, Khachaturian AS, Trzepacz P, Amatniek J, et al. Neuropsychiatric symptoms in Alzheimer's disease. Alzheimers Dement. 2011; 7: 532-539.

11. Steinberg M, Shao HB, Zandi P, Lyketsos CG, Welsh-Bohmer KA, Norton MC, et al. Point and 5year period prevalence of neuropsychiatric symptoms in dementia: The cache county study. Int J Geriatr Psychiatry. 2008; 23: 170-177.

12. Casey DA. Pharmacotherapy of neuropsychiatric symptoms of dementia. Pharmacol Ther. 2015; 40: 284-287.

13. Rabins PV, Schwartz S, Black BS, Corcoran C, Fauth E, Mielke M, et al. Predictors of progression to severe Alzheimer's disease in an incidence sample. Alzheimers Dement. 2013; 9: 204-207.

14. Volicer L. Toward better terminology of behavioral symptoms of dementia. J Am Med Dir Assoc. 2012; 13: 3-4.

15. The IPA complete guides to behavioral and psychological symptoms of dementia. Milwaukee, WI: International Psychogeriatrics Association; 2015. Available from: https://www.ipaonline.org/publications/guides-to-bpsd. 
16. Finkel SI, e Silva JC, Cohen GD, Miller S, Satorius N. Behavioral and psychological symptoms of dementia: A consensus statement on current knowledge and implications for research and treatment. Am J Geriatr Psychiatry. 1998; 6: 97-100.

17. Reus VI, Fochtmann LJ, Eyler AE, Hilty DM, Horvitz-Lennon M, Jibson MD, et al. The American Psychiatric Association practice guideline on the use of antipsychotics to treat agitation or psychosis in patients with dementia. Focus. 2017; 15: 81-84.

18. Levinson D. Medicare atypical antipsychotic drug claims for elderly nursing home residents. Department of Health and Human Services. Office of the Inspector General; 2011. OEI-07-0800150. Available from: http://psychrights.org/Issues/MedicareFraud/oei-07-08-00150.pdf.

19. National partnership to improve dementia care in nursing homes: Antipsychotic medication use data report (October 2020) [Internet]. Woodlawn, Baltimore County, Maryland: Centers for Medicare \& Medicaid Services; 2020. Available from: https://www.cms.gov/files/document/antipsychotic-medication-use-data-report-updated01242020.pdf.

20. Crystal S, Jarrin OF, Rosenthal M, Hermida R, Angell B. National partnership to improve dementia care in nursing homes campaign: State and facility strategies, impact and antipsychotic medication reduction outcomes. Innov Aging. 2020; 4: igaa018.

21. Renz SM, Boltz MP, Wagner LM, Capezuti EA, Lawrence TE. Examining the feasibility and utility of an SBAR protocol in long-term care. Geriatr Nurs. 2013; 34: 295-301.

22. Harrington C, Kovner C, Mezey M, Kayser-Jones J, Burger S, Mohler M, et al. Experts recommend minimum nurse staffing standards for nursing facilities in the United States. Gerontologist. 2000; 40: 5-16.

23. Bjerre LM, Farrell B, Hogel M, Graham L, Lemay G, McCarthy L, et al. Deprescribing antipsychotics for behavioural and psychological symptoms of dementia and insomnia: Evidence-based clinical practice guideline. Can Fam Physician. 2018; 64: 17-27.

24. Reus VI, Fochtmann LJ, Eyler AE, Hilty DM, Horvitz-Lennon M, Jibson MD, et al. The American Psychiatric Association practice guideline on the use of antipsychotics to treat agitation or psychosis in patients with dementia. Am J Psychiatry. 2016; 173: 543-546.

25. Regier NG. Problems associated with the measurement of neuropsychiatric symptoms of dementia. OBM Geriatr. 2020; 4: 18.

26. Kales HC, Gitlin LN, Lyketsos CG. Management of neuropsychiatric symptoms of dementia in clinical settings: Recommendations from a multidisciplinary expert panel. J Am Geriatr Soc. 2014; 62: 762-769.

27. Hurley AC, Volicer L, Camberg L, Ashley J, Woods D, Odenheimer G, et al. Measurement of observed agitation in patients with dementia of Alzheimer's type. J Ment Health Aging. 1999; 5: 117-132.

28. Volicer L, Bass EA, Luther SL. Agitation and resistiveness to care are two separate behavioral syndromes of dementia. J Am Med Dir Assoc. 2007; 8: 527-532.

29. Volicer L. Is aggression a common symptom of dementia? J Am Med Dir Assoc. 2008; 9: 532.

30. Cohen-Mansfield J. Instruction manual for the Cohen-Mansfield inventory (CMAI). Research Institute of the Hebrew Home of Greater Washington; 1991. Available from: https://oregonbhi.org/wp-content/uploads/2020/01/CMAl Manual.pdf. 
31. Cummings J, Mintzer J, Brodaty H, Sano M, Banerjee S, Devanand DP, et al. Agitation in cognitive disorders: International Psychogeriatric Association provisional consensus clinical and research definition. Int Psychogeriatr. 2015; 27: 7-17.

32. Volicer L, Citrome L, Volavka J. Measurement of agitation and aggression in adult and aged neuropsychiatric patients: Review of definitions and frequently used measurement scales. CNS Spec. 2017; 22: 407-414.

33. Ismail Z, Goodarzi Z. Neuropsychiatric aspects of Alzheimer's disease. Clinically significant neuropsychiatric symptoms need evidence-based treatment. Pract Neurol. 2019. Available from: $\quad$ https://practicalneurology.com/articles/2019-june/neuropsychiatric-aspects-ofalzheimers-disease.

34. Cummings JL, Mega M, Gray K, Thompson SR, Carusi DA, Gornbein J, et al. The neuropsychiatric Inventory: Comprehensive assessment of psychopathology in dementia. Neurology. 1994; 44: 2308-2314.

35. Maust DT, Kim HM, Seyfried LS, Chiang C, Kavanagh J, Schneider LS, et al. Antipsychotics, other psychotropics, and the risk of death in patients with dementia: Number needed to harm. JAMA Psychiatry. 2015; 72: 438-445.

36. Volicer L, Van der Steen JT, Frijters DH. Modifiable factors related to abusive behaviors in nursing home residents with dementia. J Am Med Dir Assoc. 2009; 10: 617-622.

37. Porsteinsson AP, Drye LT, Pollock BG, Devanand DP, Frangakis C, Ismail Z, et al. Effect of citalopram on agitation in Alzheimer disease: The CitAD randomized clinical trial. JAMA. 2014; 311: 682-691.

38. Wise EA, Rosenberg PB, Lyketsos CG, Leoutsakos JM. Time course of neuropsychiatric symptoms and cognitive diagnosis in National Alzheimer's Coordinating Centers volunteers. Alzheimers Dement. 2019; 11: 333-339.

39. Liew TM. Neuropsychiatric symptoms in cognitively normal older persons, and the association with Alzheimer's and non-Alzheimer's dementia. Alzheimers Res Ther. 2020; 12: 35.

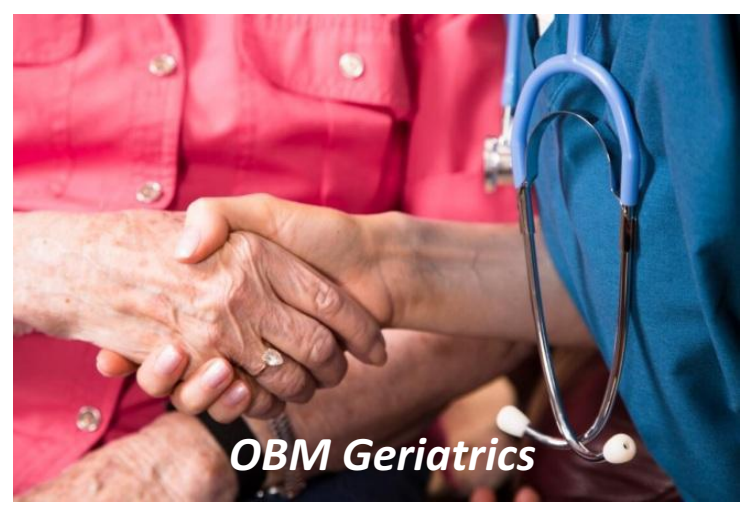

Enjoy $O B M$ Geriatrics by:

1. Submitting a manuscript

2. Joining in volunteer reviewer bank

3. Joining Editorial Board

4. Guest editing a special issue

For more details, please visit: http://www.lidsen.com/journals/geriatrics 